\title{
Effect of altered eating habits and periods during Ramadan fasting on intraocular pressure, tear secretion, corneal and anterior chamber parameters
}

H Kerimoglu, B Ozturk, K Gunduz, B Bozkurt, $\mathrm{U}$ Kamis and M Okka

\begin{abstract}
Purpose To determine whether altered eating habits and periods, especially the pre-dawn meal, during Ramadan fasting have any significant effect on intraocular pressure (IOP), tear secretion, corneal and anterior chamber parameters.

Methods IOP, basal tear secretion (BTS), reflex tear secretion (RTS), and Pentacam measurements of 31 healthy volunteers were performed at 0800 and 1600 hours during Ramadan fasting and 1 month later during non-fasting period.

Results Comparison of measurements
\end{abstract} between fasting and non-fasting periods at 0800 hours revealed significantly higher values for IOP $(P=0.005)$, RTS $(P=0.006)$, and BTS $(P=0.014)$ during fasting. Conversely at 1600 hours, IOP was significantly lower during fasting $(P=0.013)$ and no statistically significant difference was noted for RTS and BTS. IOP showed a diurnal variation of $2.45 \mathrm{mmHg}$ $(P<0.001)$ and BTS showed a $3.06 \mathrm{~mm}$ decrease $(P=0.04)$ during the fasting period. No significant differences could be found in the corneal and anterior chamber parameters during fasting and non-fasting periods.

Conclusions Our results revealed that fluid loading at the pre-dawn meal during Ramadan fasting might increase the IOP and tear secretion in the early morning period and these values decrease remarkably at the end of $12 \mathrm{~h}$ of fasting due to dehydration.

Eye (2010) 24, 97-100; doi:10.1038/eye.2009.96; published online 8 May 2009
Keywords: ramadan fasting; predawn meal; intraocular pressure; tear secretion; anterior chamber parameters; pentacam

\section{Introduction}

Religious fasting is a period of abstinence of food and drink from dawn to sunset during the month of Ramadan. The amount of food and fluid intake shows marked change due to fasting during the day and consuming food and fluid beginning after sunset and lasting until dawn. During this month, people of Islamic belief eat food and drink fluids twice between sunset and dawn; a break-of-fasting meal, just after sunset at the end of the fasting period and also either at midnight before sleep or pre-dawn meal just before dawn, which is the beginning of the new fasting period. To endure the effects of hunger and dehydration, people intentionally eat a significant amount of food and drink fluids just before dawn. In an earlier study, Prentice $e t a l^{1}$ investigated the effects of dehydration on water balance and milk synthesis in lactating women who were fasting during Ramadan and found that the daily water turnover of 6.41 in the lactating women was 21 more than that in the controls. Fasting people drink nearly $50 \%$ more amount of water than non-fasting people in a limited time period between sunset and dawn, and a significant amount of this water intake occurs at the pre-dawn meal just before the sunrise; this condition appears analogous to water loading. It is known that oral water loading transiently elevates intraocular pressure (IOP) through mechanisms that remain unexplained. ${ }^{2}$ Water loading due to changed eating habits and periods
Faculty of Medicine, Department of Ophthalmology, Selcuk University, Konya, Turkey

Correspondence: H Kerimoglu, Faculty of Medicine, Department of Ophthalmology, Selcuk University, Konya 42080, Turkey Tel: + 903322236210 ; Fax: + 903322236505

E-mail: hurkanka@ yahoo.com

Received: 21 July 2008 Accepted in revised form: 26 March 2009

Published online: 8 May 2009

This study was carried out at Faculty of Medicine, Department of Ophthalmology, Selcuk University, Konya, Turkey.

Proprietary interest: None 
may affect IOP, tear secretion, and, maybe, corneal and anterior chamber parameters.

To the best of our knowledge, there are only a few studies performed evaluating the effects of religious fasting on IOP and tear secretion in the literature, and there is no report about the anterior chamber parameters. ${ }^{3-5}$ In the study of Dadeya et $\mathrm{al}^{4}{ }^{4}$ fasting was shown to alter diurnal IOP in healthy subjects, whereas Kayikcioglu $e t \mathrm{al}^{3}$ could not find such a correlation. There is only one report evaluating the effects of fasting on basal tear secretion (BTS), which concluded that religious fasting does not seem to affect BTS in healthy individuals. ${ }^{5}$ All of these reports emphasized the effect of dehydration caused by fasting on IOP and BTS.

In this study, we aimed to determine whether changed eating habits and periods, especially the pre-dawn meal, during Ramadan fasting have any significant effect on IOP, tear secretion, corneal and anterior chamber parameters.

\section{Materials and methods}

The study included 31 healthy volunteers, who were informed about the study and signed informed consent to participate in the study. Approval for this clinical study was obtained from the Institutional Review Board/ Ethics Committee at Selcuk University, Konya, Turkey. Patients with systemic diseases such as diabetes and hypertension, and eye diseases such as ocular hypertension, glaucoma, dry eye syndrome, blepharitis, and previous ocular surgery, were excluded. Only patients who had their last meal just before dawn were included in the study for precise estimation of the fasting hours. IOP measurements were performed with Goldmann applanation tonometre and corneal and anterior chamber measurements were performed with Oculus Pentacam (OCULUS Optikgeräte $\mathrm{GmbH}$, Wetzlar, Germany). Both reflex tear secretion (RTS) and BTS were measured with and without topical anaesthetic eye drop (proparacaine hydrochloride) by using schirmer strips. Measurements were performed on the left eyes of the volunteers at 0800 and 1600 hours at approximately fourth and twelfth hours of fasting, respectively, in the order of Pentacam, RTS, BTS, and Goldmann applanation tonometre. Measurements were repeated at the same order and at the same time points 1 month after Ramadan when the subjects were on a normal diet.

Statistical analysis was performed using the SPSS program (SPSS for Windows version 11.5; SPSS Inc., Chicago, IL, USA). Parameters were assessed for normal distribution by histograms and with the KolmogorovSmirnov test. For parameters showing normal distribution, paired sample $t$-test was used to compare the parameters measured at the Ramadan month and 1 month later at 0800 and 1600 hours to assess the effect of fasting on these parameters in different hours, whereas the Wilcoxon signed rank test was used to compare the non-parametric parameters. We also compared the change in parameters between 0800 and 1600 hours during the Ramadan month and 1 month later to evaluate the diurnal variation of these parameters during fasting and non-fasting periods using paired sample $t$-test or Wilcoxon signed rank test.

\section{Results}

Twelve women and 19 men were enrolled in the study. The mean age of the volunteers was $31.7 \pm 5.3$ years (24-36 years). Mean values of IOP, RTS, BTS, corneal and anterior chamber measurements at different time points during fasting and non-fasting periods are given in Table 1, whereas diurnal variation of IOP, RTS, and BTS during fasting and non-fasting periods is presented in Table 2.

Comparison of measurements between fasting and non-fasting periods at 0800 hours revealed significantly higher values for IOP $(P=0.005)$, RTS $(P=0.006)$, and BTS $(P=0.014)$ during fasting. Conversely, at 1600 hours, IOP was significantly lower during fasting $(P=0.013)$ and no statistically significant difference was noted for RTS and BTS. IOP showed a diurnal variation of $2.45 \mathrm{mmHg}(P<0.001)$ and BTS showed a $3.06 \mathrm{~mm}$

Table 1 Intraocular pressure, reflex and basal tear secretions, and corneal and anterior chamber parameters during fasting and non-fasting periods

\begin{tabular}{|c|c|c|c|c|c|c|}
\hline & \multicolumn{2}{|c|}{0800 hours } & \multirow[t]{2}{*}{ P-value } & \multicolumn{2}{|c|}{1600 hours } & \multirow[t]{2}{*}{ P-value } \\
\hline & Fasting & Non-fasting & & Fasting & Non-fasting & \\
\hline $\mathrm{IOP}(\mathrm{mmHg})^{\mathrm{a}}$ & $14.19 \pm 3.53$ & $12.03 \pm 2.99$ & 0.005 & $11.74 \pm 2.39$ & $13.13 \pm 2.39$ & 0.013 \\
\hline $\operatorname{RTS}(\mathrm{mm} / 5 \mathrm{~min})^{\mathrm{a}}$ & $30.26 \pm 8.49$ & $25.06 \pm 9.63$ & 0.006 & $27.55 \pm 9.34$ & $27.58 \pm 9.01$ & 0.813 \\
\hline BTS $(\mathrm{mm} / 5 \mathrm{~min})^{\mathrm{a}}$ & $16.42 \pm 7.41$ & $14.00 \pm 8.21$ & 0.014 & $13.35 \pm 6.56$ & $13.61 \pm 7.49$ & 0.79 \\
\hline
\end{tabular}

BTS, basal tear secretion; IOP, intraocular pressure; RTS, reflex tear secretion.

ailcoxon signed rank test. 
Table 2 Intraday differences of intraocular pressure, reflex and basal tear secretions between 0800 and 1600 hours during fasting and non-fasting periods

\begin{tabular}{lccccc}
\hline & Fasting & & Non-fasting & P-value \\
\cline { 2 - 2 } \cline { 5 - 6 } & 0800 to 1600 hours & & 0800 to 1600 hours & \\
\hline IOP (mmHg) & $2.45 \pm 2.67$ & & $-1.10 \pm 3.28$ & $<0.001$ \\
RTS (mm/5 min) & $2.71 \pm 8.55$ & & $-2.51 \pm 8.38$ & 0.06 \\
BTS (mm/5 min) & $3.06 \pm 6.37$ & & $0.38 \pm 6.18$ & 0.04 \\
\hline
\end{tabular}

BTS, basal tear secretion; IOP, intraocular pressure; RTS, reflex tear secretion.

decrease $(P=0.04)$ during the fasting period. No significant differences could be found in the corneal and anterior chamber parameters during fasting and non-fasting periods.

\section{Discussion}

During the lunar month of Ramadan, Muslims eat only between sunset and dawn, and the change in eating habits and periods (midnight dinner and pre-dawn meal) might affect their IOP, tear secretion, and the anterior chamber parameters. The fasting period changes according to the daytime period at that month of the year because of the difference between lunar and solar calendars. Abstinence duration also changes among people within the same day. Most of the fasting Muslims have their last meal and drink a large amount of fluid just before dawn and abstain from food and fluid until sunset, whereas some of them eat their last meal at midnight dinner before going to sleep and abstain from taking food and fluid until sunset of the next day, which makes at least a $4 \mathrm{~h}$ difference in fasting duration. Therefore, we chose healthy volunteers who had their last meal just before dawn to have a uniform group.

There are only a few reports in the literature on fasting and IOP. ${ }^{3-4}$ Kayikcioglu et $a l^{3}$ showed that fasting did not alter diurnal IOP in healthy subjects. Dadeya et $a l^{4}$ measured IOP four times during the daytime beginning from 0900 to 1800 hours with $3 \mathrm{~h}$ interval and found significantly lower IOP values at all time points during fasting. In our study, the mean IOP was $14.19 \pm 3.53 \mathrm{mmHg}$ at 0800 hours and showed a decrease in the afternoon to $11.74 \pm 2.39 \mathrm{mmHg}$ at 1600 hours during fasting period, whereas a $1.1 \mathrm{mmHg}$ IOP rise was observed during the non-fasting period, which was nonsignificant. In the study group of Dadeya et al, there was a $0.1 \mathrm{mmHg}$ rise in the fasting group and $0.6 \mathrm{mmHg}$ rise in the non-fasting group between 0900 and 1500 hours. They also noticed another $1.6 \mathrm{mmHg}$ rise in $3 \mathrm{~h}$ between 1500 and 1800 hours in the non-fasting group. ${ }^{4}$

None of the above studies mentioned the eating periods (midnight dinner and pre-dawn meal) and fasting duration before the first measurement. Higher levels of IOP at 0800 hours maybe related to the changed eating habits and periods. We measured IOP at 0800 hours nearly $4 \mathrm{~h}$ after the pre-dawn meal. Most commonly, people eat carbohydrate-weighted food and take remarkable amount of fluid at the pre-dawn meal. They drink nearly 50\% more amount of water than they consume on a normal diet in $24 \mathrm{~h}$ period, and a significant amount of this is consumed at the pre-dawn meal just before the sunrise. ${ }^{1}$

There is evidence from earlier studies that drinking 11 of water increases the IOP, with a mean maximum increase of $4.4 \mathrm{mmHg}$ for more than $2 \mathrm{~h}$ in healthy eyes. ${ }^{6}$ Although we do not know the exact volume of water taken at the pre-dawn meal, water loading at the pre-dawn meal might be the cause of increased IOP in the morning. Another factor that may affect the IOP measurements is the central corneal thickness (CCT) ${ }^{7-8}$ However, our results revealed that mean CCT of our study population was within normal limits and there was no statistically significant difference of CCT between fasting and non-fasting periods.

Tear secretion during fasting period was investigated in another study of Kayikcioglu et $a l^{5}$ which found that religious fasting in the winter season did not affect BTS in healthy individuals. ${ }^{5}$ They found only a 0.2 mm decrease in BTS between 0800 and 1700 hours during the fasting period. In our study, we found significantly higher RTS and BTS values at 0800 hour measurement during fasting compared with non-fasting period, and the values decreased remarkably with a mean of $2.71 \pm 8.55$ and $3.06 \pm 6.37 \mathrm{~mm}$, respectively, at 1600 hours in the fasting group. Higher values of RTS and BTS in the morning in fasting group compared with non-fasting group may also be explained with water loading at the pre-dawn meal a few hours ago. The diurnal variation in tear secretion was significantly higher in our study, with lower values in the afternoon close to the end of an abstinence period. We performed this study in October, in a warmer climate, the level of dehydration being higher compared with the study of Kayikcioglu et al, ${ }^{5}$ performed in January.

In analysing downside to this study, we could not objectively quantify the fluid intake during the fasting and non-fasting periods and the differences in time of awakeness before IOP measurement. Although we know that these people consume a large amount of fluid before dawn we do not know the exact volume as well as the exact volume of fluid consumed during the non-fasting period on a regular diet. 
To the best of our knowledge, there is no study in the literature evaluating the effect of fasting on the corneal and anterior chamber parameters. We measured corneal and anterior chamber parameters with Oculus Pentacam, which allows fast, non-contact examination of the anterior segment of the eye. Our results revealed no significant difference between fasting and non-fasting periods and between 0800 and 1600 hours during the fasting period. We only observed a slight increase in the CCT in 0800 hour measurement in fasting period $(540.8 \pm 33.02 \mu \mathrm{m})$ compared with the afternoon measurement $(535.03 \pm 35.75 \mu \mathrm{m})$, which might also be a result of fluid loading at the pre-dawn meal, but the difference was not statistically significant.

There is new data in the recent literature on dehydration caused by Ramadan fasting and its effects on IOP and tear secretion. ${ }^{3-5}$ In these reports, the effects of changed eating habits and periods, and particularly the effect of pre-dawn meal, were neglected. Our results highlighted an important aspect of Ramadan fasting and suggested that fluid loading at the pre-dawn meal might increase the IOP and tear secretion in the early morning period. No differences could be found in the corneal and anterior chamber parameters. One important point that should be taken into consideration is the period of Ramadan month. As Islamic calender is a lunar calendar and Ramadan occurs at different times in the seasonal year over a 33-year cycle, the effect of dehydration might be more pronounced in warmer climates.

\section{References}

1 Prentice AM, Lamb WH, Prentice A, Coward WA. The effect of water abstention on milk synthesis in lactating women. Clin Sci (Lond) 1984; 66: 291-298.

2 Brucculeri M, Hammel T, Harris A, Malinovsky V, Martin B. Regulation of intraocular pressure after water drinking. J Glaucoma 1999; 8: 111-116.

3 Kayikcioglu O, Guler C. Religious fasting and intra-ocular pressure. J Glaucoma 2000; 9: 413-414.

4 Dadeya S, Kamlesh, Shibal F, Khurana C, Khanna A. Effect of religious fasting on intra-ocular pressure. Eye 2002; 16: 463-465.

5 Kayikcioglu O, Erkin EF, Erakgun T. The influence of religious fasting on basal tear secretion and tear break up time. Int Ophthalmol 1999; 22: 67-69.

6 Buckingham T, Young R. The rise and fall of intra-ocular pressure: the influence of physiological factors. Ophthalmic Physiol Opt 1986; 6: 95-99.

7 Doughty MJ, Zaman ML. Human corneal thickness and its impact on intraocular pressure measures: a review and meta-analysis approach. Surv Ophthalmol 2000; 44: 367-408.

8 Hamilton KE, Pye DC, Aggarwala S, Evian S, Khosla J, Perera R. Diurnal variation of central corneal thickness and Goldmann applanation tonometry estimates of intraocular pressure. J Glaucoma 2007; 16: 29-35. 